\title{
A SIMPLIFICATION OF THE COMPUTATION OF THE NATURAL REPRESENTATION OF THE SYMMETRIC GROUP $\boldsymbol{S}_{\boldsymbol{n}}$
}

\author{
JOSEPH M. CLIFTON
}

\begin{abstract}
Recent use of the symmetric group $S_{n}$ in processing identities in nonassociative algebras has brought a renewed interest in the natural (integral) irreducible representation of $S_{n}$ [3]. Using a construction due to A. Young, $\mathrm{H}$. Boerner gives a prescription for writing down the natural (integral) irreducible representation of the symmetric group $S_{n}$ over an algebraically closed field of characteristic zero [1, p. 119]. Writing down the matrices using this prescription is rather tedious, and becomes computationally impossible for large $n(n>9)$ because of the need to calculate chains. In this paper, we simplify the computation of these matrices by eliminating the need to calculate chains. The calculation of the chains is replaced by the simple act of setting up and inverting an upper triangular matrix $A_{\boldsymbol{I}}$.
\end{abstract}

Let $n$ be a fixed positive integer. By a frame, we will mean $n$ squares arranged in rows of decreasing length such that the left-hand edges of the rows coincide. A tableau is obtained by placing the numbers 1 through $n$ in the squares of a frame. For a given frame, let $[\pi]$ denote the matrix corresponding to the natural (integral) irreducible representation (over an algebraically closed field of characteristic zero) of $\pi \in S_{n}$. For each $\pi \in S_{n}$, we show how to construct a matrix $A_{\pi}$ such that [ $\pi]=A_{I}^{-1} A_{n}$, where $I$ is the identity permutation in $S_{n}$.

Throughout the remainder of this paper, we will assume that all tableaux belong to a given frame of $n$ squares. We will also assume that the irreducible representation of $S_{n}$ corresponding to this frame is over $F$, an algebraically closed field of characteristic zero, and that the degree of this representation is $f$.

Given a tableau $T$, let $P$ denote the product of the positive symmetric groups of the rows of $T$, and let $N$ denote the product of the negative symmetric groups of the columns of $T[5$, p. 391]. Let $e=f / n ! P N$. Then $e$ is a nonzero idempotent in the group algebra of $S_{n}$ over $F[1, \mathrm{p}$. 109], which we will call the idempotent corresponding to $T$.

Let $T_{1}, T_{2}, \ldots, T_{n}$ ! be the distinct tableaux for our given frame, and let $e_{1}, e_{2}, \ldots, e_{n !}$ be the corresponding idempotents. For $i, j=1,2, \ldots, n !$, let $s_{i j}$ denote the permutation in $S_{n}$ which transforms $T_{j}$ into $T_{i}$. It is clear that $s_{i j}^{-1}=s_{j i}$ and $s_{i j} s_{j k}=s_{i k}$. Also, $e_{i}=s_{i j} e_{j} s_{j i}[1, \mathrm{p} .105]$.

For $i, j=1,2, \ldots, n !$, we define numbers $\varepsilon_{i j}$ as follows:

If there exists two numbers in one column of $T_{i}$ and in one row of $T_{j}$, then we set $\varepsilon_{i j}=0$. If not, then there exists a vertical permuta-

Received by the editors January 14, 1981.

1980 Mathematics Subject Classification. Primary 20C30; Secondary $20 \mathrm{C10}$.

(c) 1981 American Mathematical Society $0002-9939 / 81 / 0000-0455 / \$ 01.75$ 
tion $q$ for $T_{i}$ which leaves the columns of $T_{i}$ fixed as sets and takes the numbers of $T_{i}$ into the correct rows they occupy in $T_{j}[1, \mathrm{pp}$. 106-108]. In this case, we set $\varepsilon_{i j}=\operatorname{sgn}(q)$.

Then we have $e_{i} e_{j}=\varepsilon_{i j} s_{i j} e_{j}$ for $i, j=1,2, \ldots, n ![4$, p. 22].

Let $\pi \in S_{n}$ and $1 \leqslant i, j \leqslant n$ !. Then $\pi T_{j}=T_{r}$ for some $r$, so $\pi=s_{r j}$. Therefore, $e_{i} \pi e_{j}=e_{i} s_{r j} e_{j}=e_{i} e_{r} s_{r j}=\varepsilon_{i r} s_{i r} e_{r} s_{r j}=\varepsilon_{i r} s_{i j} e_{j}$. Denote $\varepsilon_{i r}$ by $\varepsilon_{i j}^{\pi}$. Then $e_{i} \pi e_{j}=\varepsilon_{i j}^{\pi} s_{i j} e_{j}$ for each $\pi \in S_{n}$ and for each $i, j=1,2, \ldots, n$ !.

Let $T_{1}, T_{2}, \ldots, T_{f}$ denote the standard tableaux in dictionary order [1, p. 112]. For each $\pi \in S_{n}$, form the $f \times f$ matrix $A_{\pi}$ by letting $\left[A_{\pi}\right]_{i j}=\varepsilon_{i j}^{\pi}$ for $i, j=$ $1,2, \ldots, f$. Thus, the $i, j$ entry of $A_{\pi}$ can be calculated as follows:

Apply $\pi$ to the tableau $T_{j}$. If there exist two numbers that appear together in a column of $T_{i}$ and a row of $\pi T_{j}$, then $\left[A_{\pi}\right]_{i j}=0$. If not, then $\left[A_{\pi}\right]_{i j}$ equals the sign of the vertical permutation for $T_{i}$ which leaves the columns of $T_{i}$ fixed as sets and takes the numbers of $T_{i}$ into the correct rows they occupy in $\pi T_{j}$.

THEOREM. For each $\pi \in S_{n},[\pi]=A_{I}^{-1} A_{\pi}$, where $I$ is the identity permutation of $S_{n}$.

Proof. $A_{I}$ is upper triangular with $\left[A_{I}\right]_{i i}=+1$ for $i=1,2, \ldots, f[1$, pp. 109 and 113]. Thus, $A_{I}$ has determinant +1 , and hence is invertible. Let $w_{i j}=\left[A_{I}^{-1}\right]_{i j}$, $i, j=1, \ldots, f$. If we denote the natural units [4, p. 51] by $g_{i j}$, then $g_{i j}=$ $\sum_{k=1}^{f} s_{i k} e_{k} w_{j k}\left[4\right.$, p. 53], and $g_{i j} g_{k m}=\delta_{j k} g_{i m}\left[4\right.$, p. 53]. Now $[\pi]_{i j} g_{i j}=g_{i i} \pi g_{j j}[1, p$. 117]. We compute

$$
\begin{aligned}
g_{i i} \pi g_{j j} & =\left(\sum_{k=1}^{f} s_{i k} e_{k} w_{i k}\right) \pi\left(\sum_{m=1}^{f} s_{j m} e_{m} w_{j m}\right) \\
& =\sum_{k, m=1}^{f} w_{i k} s_{i k} e_{k} \pi e_{j} s_{j m} w_{j m}=\sum_{k, m=1}^{f} w_{i k} s_{i k} \varepsilon_{k j}^{\pi} s_{k j} e_{j} s_{j m} w_{j m} \\
& =\left(\sum_{k=1}^{f} w_{i k} \varepsilon_{k j}^{\pi}\right)\left(\sum_{m=1}^{f} s_{i m} e_{m} w_{j m}\right)=\left(\sum_{k=1}^{f} w_{i k} \varepsilon_{k j}^{\pi}\right) g_{i j} .
\end{aligned}
$$

Therefore, $[\pi]_{i j}=\sum_{k=1}^{f} w_{i k} \varepsilon_{k j}^{\pi}$. Thus, $[\pi]=A_{I}^{-1} A_{\pi}$.

Note that we get an equivalent representation of $S_{n}$ for any set of $f$ tableaux (not necessarily standard) such that $A_{I}$ is invertible. If the determinant of $A_{I}$ is +1 or -1 , then the representation of $S_{n}$ will be integral. In [2], the possibility of choosing $f$ tableaux (not necessarily standard) such that $A_{I}$ is the identity matrix is considered. For frames which this is possible, we have that $\left\{A_{\pi}: \pi \in S_{n}\right\}$ is an easily computed irreducible representation of $S_{n}$ in which the entries of all the matrices are restricted to $+1,-1$, or 0 . Other advantages to such a set of tableaux are listed in [2].

As a final note, we mention that the first footnote in [4, p. 53] should read "**Young IV, p. 258". Also, the " $r$ " and " $s$ " are interchanged in the next to last line in [5, p. 401]. Finally, the second and third lines in [5, p. 402] (Young's fourth paper 
On quantitative substitutional analysis) are in error. To see this, consider the frame $(3,2)$. Let $X_{r s}=\delta_{r 5} \delta_{s 5}=Y_{r s}, r, s=1,2, \ldots, 5$. Since $M_{5}=I, \Sigma X_{r s} P_{r} \sigma_{r s} N_{s} M_{s}=$ $\sum Y_{r s} P_{r} \sigma_{r s} N_{s}$. Now $E-S=A_{I}^{T}$. Thus $\left[Y_{r s}\right]=\left[X_{r s}\right] \neq\left[X_{r s}\right](E-S)$. One can show that the third line in $\left[5\right.$, p. 402] should real " $\left[X_{r s}\right]=\left[Y_{r s}\right](E-S)^{T}$ ".

ACKNOWLEDGEMENT. The material for this paper was taken from my thesis [2] under the direction of Professor Irvin R. Hentzel at Iowa State University.

\section{REFERENCES}

1. H. Boerner, Representations of groups with special consideration for the needs of modern physics, North-Holland, Amsterdam, 1963.

2. J. Clifton, Complete sets of orthogonal tableaux, Ph. D. Dissertation, Iowa State University, Ames, Iowa, 1980.

3. I. Hentzel, Processing identities by group representation, Computers in Non-associative Rings and Algebras (R. E. Beck and B. Kolman, Eds.), Academic Press, New York, 1977, pp. 13-40.

4. D. Rutherford, Substitutional analysis, The Edinburgh Univ. Press, Edinburgh, 1948.

5. A. Young, The collected papers of Alfred Young, Univ. Toronto Press, Toronto, 1977.

Bell LABoratorits, NAPERVILLE-WheATON ROAD, NAPERVILLE, ILLINOIS 60566 